\title{
Бесконечнозначная логика Лукасевича и критерии наличия фактор-семантики у многозначных ЛОГИК
}

\author{
Н. Н. ПРЕЛОВСКИЙ
}

\begin{abstract}
The article deals with a special kind of bivalent semantics for multi-valued logics, called factor-semantics. A concept of local factor-semantics is introduced while constructing matrices $\mathfrak{K}$ and $\mathfrak{K}^{\prime}$, isomorphic to the standard rational-valued matrix for infinite-valued logic of Łukasiewicz.
\end{abstract}

Keywords: logics of Łukasiewicz, factor-semantics, logical matrices, isomorphism, local factor-semantics

А.С. Карпенко (см. [1, с. 311-315]) описывает построение особого впервые рассмотренного им в [3] типа бивалентных семантик для различных многозначных логических систем, включающих конечнозначные логики Лукасевича $\mathrm{E}_{n}$ и конечнозначные логики Клини $K_{n}$. Данный тип бивалентных семантик получил название «фактор-семантик».

Суть фактор-семантики в первом приближении сводится к отказу от интерпретации пропозициональных логических формул неклассическими логическими значениями, рассматриваемыми в качестве не имеющих внутренней структуры элементов соответствующей алгебры, и переходу к интерпретации формул на множестве классов, образованных конечными или бесконечными последовательностями классических истинностных значений $T$ и $F$ (в дальнейшем - $T F$-последовательностями).

Несмотря на внесение столь радикальных изменений в определения семантических понятий, А.С. Карпенко доказал, что 
получающиеся в результате всех преобразований структуры изоморфны исходным логическим матрицам, являющимся характеристическими для соответствующих логик. Образующие фактор-семантики логические матрицы в дальнейшем часто будут называться фактор-матрицами, в отличие от исходных характеристических матриц.

Изоморфизм двух объектов является очень сильным свойством, и во многих контекстах может означать математическую неразличимость практически «всех» свойств этих объектов. В случае конечнозначных логических матриц, например, изоморфизм матриц $\mathfrak{M}_{1}$ и $\mathfrak{M}_{2}$ (символически будем обозначать этот факт выражением $\mathfrak{M}_{1} \equiv \mathfrak{M}_{2}$ ) гарантирует совпадение классов тавтологий $E\left(\mathfrak{M}_{1}\right)$ и $E\left(\mathfrak{M}_{2}\right)$ в этих матрицах, а также совпадение соответствующих этим матрицам логических систем, полученных добавлением к обеим матрицам того или иного определения логического следования. Между множествами-носителями $M_{1}$ и $M_{2}$ этих матриц может быть установлено взаимнооднозначное соответствие $\varphi: M_{1} \mapsto M_{2}$, гарантирующее выполнение равенства $\left|M_{1}\right|=\left|M_{2}\right|$, а также совпадение табличных определений соответствующих связок с точностью до переименования элементов множеств-носителей. Таким образом, отвлечение от способов задания изоморфных объектов (в данном случае придется отвлечься от внутренней структуры элементов матриц, соответствующих фактор-семантикам) ведет к невозможности их практического различения.

Но такая обусловленная изоморфизмом неразличимость сохраняется лишь при рассмотрении фактор-семантик в узком контексте логик, для которых фактор-семантики уже построены и наличие отношения изоморфизма (что эквивалентно наличию сохраняющего связки взаимнооднозначного отображения $\varphi)$ между соответствующими матрицами уже доказано.

Возникает вопрос о том, какие многозначные логики могут иметь фактор-семантики, а какие - нет. Всегда ли возможно построение фактор-семантики, изоморфной исходной логической матрице, или же в некоторых случаях изоморфизм является слишком большой «роскошью», и тогда свойства соответствующей фактор-семантике матрицы (в некотором смысле, такая 
матрица, конечно, уже не может считаться точной семантикой для заданной исходной матрицей логики) могут совпадать лишь с некоторыми из свойств исходной матрицы, а с другими ее свойствами могут и не совпадать? Или же в некоторых случаях возможно добиться изоморфизма с исходными матрицами, но за счет сохранения лишь отдельных черт фактор-семантики и одновременной утраты других ее черт?

Ответ на первый из этих вопросов требует выявления логических матриц, для которых не могут быть построены факторсемантики, и такие матрицы существуют. В частности, в [1] отмечается, что циклическое отрицание логик Поста $P_{n}$ не может быть выражено через покомпонентные булевы операции. С другой стороны, для любой конечнозначной логики процесс построения соответствующей фактор-семантики, если она существует, может быть алгоритмизирован. Для этого необходимо и достаточно рассмотреть множество всех отношений $R(\alpha, \beta)$ между особым образом заданными $T F$-последовательностями $\alpha$ и $\beta$ из всевозможных классов эквивалентности $[\alpha]$ и $[\beta]$ (это возможно с учетом конечности множеств всех бинарных отношений и всех отношений эквивалентности, заданных на конечном множестве). В случае бесконечнозначной логики, ситуация изменяется, рассмотрению некоторых предварительных результатов в этом направлении и посвящена данная статья.

Приведем формальное определение фактор-семантик на примере $n$-значных логик Лукасевича $\mathrm{E}_{n}$.

С булевой алгеброй

$$
\mathcal{A}^{B}=<B^{s}, \neg^{+}, \supset^{+}>
$$

ассоциируется логическая матрица

$$
\mathfrak{M}_{s}^{C}=<B^{s}, \neg^{+}, \supset^{+},\left\{T^{s}\right\}>.
$$

Эта логическая матрица представляет собой $s$-кратное прямое произведение двузначной характеристической для классической логики матрицы

$$
\mathfrak{M}_{2}^{C}=<\{T, F\}, \neg, \supset,\{T\}>
$$


на саму себя (то есть декартову $s$-ю степень данной матрицы).

Таким образом, любой элемент $\alpha \in B^{s}$ есть последовательность компонентов $T$ и $F$, состоящая из $s$ вхождений этих компонентов, то есть $\alpha$ есть $<a_{1}, \ldots, a_{s}>$, где $\forall i \leq s\left(a_{i} \in\{T, F\}\right)$.

Через $\eta(\alpha)$ обозначается число компонентов $\alpha$, paвных $T$. Определим на элементах $B^{s}$ следующее отношение эквивалентности. Так, для всяких $\alpha, \beta$ в $B^{s}$ будем говорить, что $\alpha \cong \beta$, если $\eta(\alpha)=\eta(\beta)$. Рассмотрим фактор-множество $B^{s} / \cong$ множества $B^{s}$ по отношению $\cong$. Очевидно, что

$$
\left|B^{s} / \cong\right|=s+1
$$

Если $\alpha \in B^{s}$, обозначим, соответствующий $\alpha$ класс эквивалентности через $[\alpha]$. На множестве $B^{s} / \cong$ определим операции $\neg \mathfrak{£}_{s+1}$ и $\rightarrow \mathrm{£}_{s+1}$ :

$$
\begin{aligned}
\neg^{\mathrm{E}_{s+1}}[\alpha] & =\left[\neg^{+} \alpha\right], \\
{[\alpha] \rightarrow^{\mathrm{E}_{s+1}}[\beta] } & =\left[\alpha^{\prime} \supset^{+} \beta^{\prime}\right],
\end{aligned}
$$

где $\alpha, \beta \in B^{s} / \cong, \alpha^{\prime} \in[\alpha], \beta^{\prime} \in[\beta]$ и $R\left(\alpha^{\prime}, \beta^{\prime}\right)$, причем отношение $R$ определяется следующим образом:

$$
R\left(<a_{1}, \ldots, a_{s}>,<b_{1}, \ldots, b_{s}>\right)
$$

имеет место, если и только если выполняется одно из следующих условий:

1. $\forall i \leq s\left(a_{i}=T \Rightarrow b_{i}=T\right)$, если $\eta(\alpha) \leq \eta(\beta)$;

2. $\forall i \leq s\left(b_{i}=T \Rightarrow a_{i}=T\right)$, если $\eta(\alpha)>\eta(\beta)$.

Таким образом, получена матрица

$$
\mathfrak{N}_{s+1}^{\mathrm{E}}=<B^{s} / \cong, \neg^{\mathrm{E}_{s+1}}, \rightarrow^{\mathrm{E}_{s+1}},\left\{\left[T^{s}\right]\right\}>.
$$

Данная матрица, согласно теореме 3 (см. [1, с. 312]), изоморфна характеристической для $n$-значной логики Лукасевича $\mathrm{E}_{n}$ матрице:

$$
\mathfrak{M}_{n}^{\mathrm{E}}=<V_{n}, \sim, \rightarrow,\{1\}>
$$


при $n=s+1$, где $V_{n}=\left\{0, \frac{1}{n}, \ldots, \frac{n-1}{n}, 1\right\}$, а для всех $v, v_{1}, v_{2} \in$ $V_{n}$ выполняется $\sim v=1-v$, и $v_{1} \rightarrow v_{2}=1$, если $v_{1} \leq v_{2} ;$ в противном случае: $v_{1} \rightarrow v_{2}=1-v_{1}+v_{2}$.

Возникает вопрос, можно ли построить аналогичным образом фактор-семантику для бесконечнозначной логики Лукасевича $\mathrm{E}_{\infty}$

Отметим, что определение матрицы $\mathfrak{M}_{\infty}^{\mathrm{L}}$, являющейся характеристической для бесконечнозначной логики Лукасевича, отличается от определения матрицы $\mathfrak{M}_{n}^{\complement}$ лишь тем, что множество $V_{n}$ заменяется на множество всех рациональных или действительных чисел в интервале [0;1], а определения соответствующих функций остаются неизменными с поправкой на новое множество значений. Выбор рациональнозначного или действительнозначного интервала во многих контекстах оказывается несущественным, поскольку класс тавтологий $\mathrm{E}_{\infty}$ является инвариантным относительно данного выбора.При переходе к рассмотрению фактор-семантики бесконечнозначной логики Лукасевича это различение приобретает значимый характер.

А.С. Карпенко была построена фактор-матрица, являющаяся характеристической для бесконечнозначной логики $\mathrm{E}_{\Sigma}$ (см. [2, с. 269-272] и [1, с. 315-318], множество тавтологий которой представляет собой расширение множества тавтологий логики $\mathrm{Ł}_{\infty}$. Ниже будет осуществлено построение матриц, одна из которых совпадает с $\mathrm{E}_{\infty}$ по классу тавтологий, а две других изоморфны счетной характеристической матрице $\mathrm{E}_{\infty}$, то есть матрице $\mathfrak{M}_{\infty}^{£}$, множество-носитель которой представляет собой множество всех рациональных чисел из интервала $[0 ; 1]$.

С этой целью рассмотрим логические матрицы:

$\mathfrak{M}_{1}=<M_{1}, f_{1}, \ldots, f_{n}, D_{1}>$ и $\mathfrak{M}_{2}=<M_{2}, g_{1}, \ldots, g_{n}, D_{2}>$.

В дальнейшем будем считать, что соответствующие матрицы имеют одинаковую сигнатуру.

Произведением матриц $\mathfrak{M}_{1}$ и $\mathfrak{M}_{2}$ будем называть логическую матрицу

$$
\mathfrak{M}_{1} \otimes \mathfrak{M}_{2}=<M_{1} \times M_{2}, F_{1}, \ldots, F_{n}, D_{1} \times D_{2}>
$$


где «Х» означает декартово произведение соответствующих множеств, а $F_{1}, \ldots, F_{n}$ - следующим образом определенные функции:

$$
\begin{gathered}
\forall m_{1}, \ldots, m_{i} \in M_{1}, k_{1}, \ldots, k_{i} \in M_{2}, j \in\{1, \ldots, n\} \\
F_{j}\left(<m_{1}, k_{1}>, \ldots,<m_{i}, k_{i}>\right)=<f_{j}\left(m_{1}, \ldots, m_{i}\right), g_{j}\left(k_{1}, \ldots, k_{i}\right)>.
\end{gathered}
$$

Хорошо известен результат, согласно которому множество тавтологий бесконечнозначной логики Лукасевича является пересечением множеств тавтологий конечнозначных логик Лукасевича, то есть:

$$
E\left(\mathfrak{M}_{\infty}^{\mathrm{E}}\right)=\bigcap_{1}^{\infty} E\left(\mathfrak{M}_{i+1}^{\mathrm{E}}\right) .
$$

Операция произведения матриц является в логическом инструментарии аналогом теоретико-множественного пересечения множеств тавтологий, поскольку, как показал Н. Решер в [4]:

$$
E\left(\mathfrak{M}_{1} \otimes \mathfrak{M}_{2}\right)=E\left(\mathfrak{M}_{1}\right) \cap E\left(\mathfrak{M}_{2}\right)
$$

Так как операция произведения матриц и результат Н. Решера тривиально обобщаются на случай счетного множества матриц, можно сделать следующее заключение:

$$
E\left(\mathfrak{M}_{\infty}^{\mathrm{E}}\right)=E\left(\bigotimes_{1}^{\infty} \mathfrak{N}_{i+1}^{\mathrm{E}}\right)
$$

Значит, произведение всех фактор-матриц для конечнозначных логик Лукасевича в силу их изоморфизма с исходными матрицами соответствующих логик даст матрицу, совпадающую по классу тавтологий с бесконечнозначной логикой Лукасевича.

Эта матрица имеет следующий вид:

$$
\bigotimes_{1}^{\infty} \mathfrak{N}_{i+1}^{\mathrm{L}}=
$$

$$
\left\langle\left\{<\left[\alpha_{1}\right],\left[\alpha_{2}\right], \ldots>:\left[\alpha_{i}\right] \in B^{i} / \cong\right\}, \neg \underset{\otimes}{\mathrm{E}}, \rightarrow \underset{\otimes}{\mathrm{E}},\{<[T],[T T],[T T T], \ldots>\}\right\rangle,
$$




$$
\begin{gathered}
\text { где } \neg_{\otimes}^{\mathrm{E}}\left(<\left[\alpha_{1}\right],\left[\alpha_{2}\right], \ldots>\right)=<\neg^{\mathrm{E}_{2}}\left(\left[\alpha_{1}\right]\right), \neg^{\mathrm{E}_{3}}\left(\left[\alpha_{2}\right]\right), \ldots>, \mathrm{a} \\
\quad<\left[\alpha_{1}\right],\left[\alpha_{2}\right], \ldots>\rightarrow \rightarrow_{\otimes}^{\mathrm{E}}<\left[\beta_{1}\right],\left[\beta_{2}\right], \ldots>= \\
<\left[\alpha_{1}\right] \rightarrow^{\mathrm{E}_{2}}\left[\beta_{1}\right],\left[\alpha_{2}\right] \rightarrow^{\mathrm{E}_{3}}\left[\beta_{2}\right], \ldots>.
\end{gathered}
$$

Однако данная матрица, по всей видимости, в силу приводимых ниже причин не может использоваться в построении искомой фактор-семантики для бесконечнозначной логики Лукасевича.

Рассмотрим отношение эквивалентности между элементами $<\left[\alpha_{1}\right],\left[\alpha_{2}\right], \ldots>$ и $<\left[\beta_{1}\right],\left[\beta_{2}\right], \ldots>$, принадлежащими множествуносителю данной матрицы: $<\left[\alpha_{1}\right],\left[\alpha_{2}\right], \ldots>\cong<\left[\beta_{1}\right],\left[\beta_{2}\right], \ldots>\Leftrightarrow$ $\forall i \in \mathbb{N}\left(\eta\left(\alpha_{i}\right)=\eta\left(\beta_{i}\right)\right)$.

Это отношение представляется наиболее интуитивно приемлемым кандидатом на роль отношения эквивалентности, порождающего соответствующее разбиение множества элементов матрицы.

Эквивалентными в этом случае являются, например, элементы $<[T],[T F],[T F T], \ldots>$ и $<[T],[F T],[F T T], \ldots>$, если положить, что каждая, начиная со второй, из бесконечного числа $T F$-последовательностей в соответствующем компоненте каждого из элементов содержит в точности по одному вхождению $F$. То есть элементы рассматриваемой матрицы эквивалентны, если и только если они содержат в точности одни и те же классы эквивалентности на каждой из кортежных позиций. Полученное таким образом разбиение на классы является тривиальным, поскольку каждый элемент эквивалентен лишь самому себе с точностью до выбора представителя для обозначения «внутреннего» класса эквивалентности на соответствующей кортежной позиции. А именно, выполняется:

$$
\bigotimes_{1}^{\infty} \mathfrak{N}_{i+1}^{\mathrm{E}} / \cong \equiv \bigotimes_{1}^{\infty} \mathfrak{N}_{i+1}^{\mathrm{E}} .
$$

Таким образом, можно заключить, что операция произведения фактор-матриц не сохраняет свойство «быть факторматрицей». 
Однако компоненты элементов соответствующих матриц не обязательно должны представлять собой классы эквивалентности. Рассмотрим множество

$$
B^{\infty}=B \times B^{2} \times B^{3} \times \ldots
$$

$B^{\infty}$ представляет собой множество всех бесконечных последовательностей, состоящих из всевозможных конечных

$T F$-последовательностей. Мощность множества $B^{\infty}$ есть континуум, и в связи с этим оно является «слишком большим», чтобы определить на нем счетную фактор-матрицу для бесконечнозначной логики Лукасевича. Будем считать, что каждая конечная $T F$-последовательность внутри элементов множества $B^{\infty}$ сопоставлена весу $W$ этой последовательности, определенному для всякого $s$ и для всякой последовательности $<a_{1}, \ldots, a_{s}>\in B^{s}$ следующим образом:

$$
W\left(<a_{1}, \ldots, a_{s}>\right)=\frac{\eta\left(<a_{1}, \ldots, a_{s}>\right)}{s} .
$$

Рассмотрим все такие суммы $\Sigma_{1}^{\infty} W\left(<a_{1}, \ldots, a_{i}>\right)$, где $1 \leq$ $i<\omega$, a $<a_{1}, \ldots, a_{i}>-i$-е $T F$-последовательности в некотором $\alpha \in B^{\infty}$. С их помощью определим множество $B_{*}^{\infty} \subset B^{\infty}$ такое, что: $\forall \alpha \in B^{\infty}$ выполняется $\alpha \in B_{*}^{\infty}$, если $\Sigma_{1}^{\infty} W\left(<a_{1}, \ldots, a_{i}>\right)$ находится в интервале [0; 1] и является неотрицательным рациональным числом, то есть может быть выражено дробью вида $\frac{m}{n}$, где $m$ - неотрицательное целое число, $n$ - натуральное число, a $<a_{1}, \ldots, a_{i}>\in \alpha$ для всякого $i \in\{1,2, \ldots\}$.

Установим на множестве $B_{*}^{\infty}$ отношение эквивалентности таким образом, что для всяких $\alpha$ и $\beta$ из $B_{*}^{\infty}$ выполняется, что $\alpha \cong \beta$, если и только если

$$
\Sigma_{1}^{\infty} W\left(<a_{1}, \ldots, a_{i}>\right)=\Sigma_{1}^{\infty} W\left(<b_{1}, \ldots, b_{j}>\right)
$$

где $i$ и $j$ пробегают по всем конечным $T F$-последовательностям в $\alpha$ и $\beta$, соответственно.

Определим матрицу

$$
\left.\left.\mathfrak{K}=\left\langle B_{*}^{\infty} / \cong, \neg^{\mathfrak{K}}, \rightarrow^{\mathfrak{K}},\{[<<T\rangle,\langle F F\rangle,\langle F F F\rangle, \ldots\rangle\right]\right\}\right\rangle,
$$


где $B_{*}^{\infty} / \cong$ есть результат разбиения множества $B_{*}^{\infty}$ по отношению эквивалентности $\cong,[<<T>,<F F>,\langle F F F\rangle, \ldots>]$ есть класс элементов множества $B_{*}^{\infty}$ (то есть элемент множества $\left.B_{*}^{\infty} / \cong\right)$, эквивалентных последовательности $<<T>,<$ $F F>,<F F F>, \ldots>$, в которой первое вхождение $T$ является единственным, то есть класс всех таких последовательностей $\alpha=<<a_{11}>,<a_{21}, a_{22}>,<a_{31}, a_{32}, a_{33}>, \ldots>$ из $B_{*}^{\infty}$, что $\Sigma_{1}^{\infty} W\left(<a_{i 1}, \ldots, a_{i i}>\right)=1$.

Связки $\neg^{\mathfrak{K}}$ и $\rightarrow^{\mathfrak{K}}$ определяются следующим образом:

$$
\begin{gathered}
\neg^{\mathfrak{K}}\left(\left[<<a_{11}>,<a_{21}, a_{22}>,<a_{31}, a_{32}, a_{33}>, \ldots>\right]\right)= \\
{\left[<<a_{11}^{\prime}>,<a_{21}^{\prime}, a_{22}^{\prime}>, \ldots, \neg^{+}<a_{i 1}^{\prime}, \ldots, a_{i i}^{\prime}>, \ldots>\right],}
\end{gathered}
$$

где правая часть равенства представляет собой класс последовательностей, эквивалентных последовательности, все компоненты которой, кроме $\neg^{+}<a_{i 1}^{\prime}, \ldots, a_{i i}^{\prime}>$, состоят только из вхождений $F$ (в частности, если $i \neq 2$, то $<a_{21}^{\prime}, a_{22}^{\prime}>=<F F>$ ), a $<a_{i 1}^{\prime}, \ldots, a_{i i}^{\prime}>$ есть такой компонент, что:

$$
\begin{gathered}
W\left(<a_{i 1}^{\prime}, \ldots, a_{i i}^{\prime}>\right)= \\
W\left(<a_{11}>\right)+W\left(<a_{21}, a_{22}>\right)+W\left(<a_{31}, a_{32}, a_{33}>\right)+\ldots= \\
\frac{\eta\left(<a_{i 1}^{\prime}, \ldots, a_{i i}^{\prime}>\right)}{i} .
\end{gathered}
$$

$$
\begin{gathered}
{\left[<<a_{11}>,<a_{21}, a_{22}>,<a_{31}, a_{32}, a_{33}>, \ldots>\right] \rightarrow^{\mathfrak{K}}} \\
{\left[<<b_{11}>,<b_{21}, b_{22}>,<b_{31}, b_{32}, b_{33}>, \ldots>\right]=} \\
{\left[<<a_{11}^{\prime}>,<a_{21}^{\prime}, a_{22}^{\prime}>, \ldots,\right.} \\
\left.<a_{i 1}^{\prime}, \ldots, a_{i i}^{\prime}>\supset^{+}<b_{i 1}^{\prime}, \ldots, b_{i i}^{\prime}>, \ldots>\right],
\end{gathered}
$$

где правая часть равенства представляет класс последовательностей, эквивалентных последовательности, все компоненты

$$
<a_{11}^{\prime}>,<a_{21}^{\prime}, a_{22}^{\prime}>, \ldots
$$




$$
<a_{(i-1), 1}^{\prime}, \ldots, a_{(i-1),(i-1)}^{\prime}>,<a_{(i+1), 1}^{\prime}, \ldots, a_{(i+1),(i+1)}^{\prime}>, \ldots
$$

которой состоят лишь из вхождений $F$, а $<a_{i 1}^{\prime}, \ldots, a_{i i}^{\prime}>\supset^{+}<b_{i 1}^{\prime}, \ldots, b_{i i}^{\prime}>$ есть такой компонент, что:

$$
\begin{gathered}
W\left(<a_{i 1}^{\prime}, \ldots, a_{i i}^{\prime}>\right)= \\
W\left(<a_{11}>\right)+W\left(<a_{21}, a_{22}>\right)+W\left(<a_{31}, a_{32}, a_{33}>\right)+\ldots= \\
\frac{\eta\left(<a_{i 1}^{\prime}, \ldots, a_{i i}^{\prime}>\right)}{i}
\end{gathered}
$$

и

$$
W\left(<b_{i 1}^{\prime}, \ldots, b_{i i}^{\prime}>\right)=
$$$$
W\left(<b_{11}>\right)+W\left(<b_{21}, b_{22}>\right)+W\left(<b_{31}, b_{32}, b_{33}>\right)+\ldots=
$$$$
\frac{\eta\left(<b_{i 1}^{\prime}, \ldots, b_{i i}^{\prime}>\right)}{i} \text {; }
$$

и в соответствующей конечнозначной фактор-матрице $\mathfrak{N}_{i+1}^{\mathrm{E}}$ имеет место:

$$
R\left(<a_{i 1}^{\prime}, \ldots, a_{i i}^{\prime}>,<b_{i 1}^{\prime}, \ldots, b_{i i}^{\prime}>\right) .
$$

Матрица $\mathfrak{K}$ изоморфна счетной матрице $\mathfrak{M}_{\infty}^{£}$, являющейся характеристической для бесконечнозначной логики Лукасевича $\mathrm{E}_{\infty}$. Доказательство этого утверждения существенно опирается на изоморфизм между конечнозначными фактор-матрицами $\mathfrak{N}_{s+1}^{\mathrm{E}}$ и $n$-значными матрицами логик Лукасевича $\mathrm{\complement}_{n}$, в которых $n=s+1$. Докажем соответствующую теорему.

Tеорема 1. Матрица $\mathfrak{K}$ изоморфна матрице $\mathfrak{M}_{\infty}^{L}=\langle[0 ; 1], \sim, \rightarrow,\{1\}\rangle$, где $[0 ; 1] \in \mathcal{P}(\mathbb{Q})$.

ДокАЗАТЕЛЬСтво. Определим отображение $\varphi: B_{*}^{\infty} / \cong \mapsto[0 ; 1]$, что для всякого $[\alpha]$ в $B_{*}^{\infty} / \cong$ выполняется:

$$
\varphi([\alpha])=\Sigma_{1}^{\infty} W\left(<a_{i 1}, \ldots, a_{i i}>\right),
$$

где $i$ пробегает по индексам в соответствующих конечных последовательностях из $\alpha$. Данное отображение в силу определения множества $B_{*}^{\infty} / \cong$ является взаимнооднозначным. Покажем теперь, что изоморфизм имеет место, то есть: 
- $\varphi\left(\neg^{\mathfrak{K}}([\alpha])\right)=\sim \varphi([\alpha])$;

- $\varphi\left([\alpha] \rightarrow^{\mathfrak{K}}[\beta]\right)=\varphi([\alpha]) \rightarrow \varphi([\beta])$.

Рассмотрим первое равенство. Допустим, что $[\alpha]$ есть $\left[<<a_{11}>,<a_{21}, a_{22}>,<a_{31}, a_{32}, a_{33}>, \ldots>\right]$. Тогда, по определению, выполняется следующее равенство:

$$
\neg^{\mathfrak{K}}([\alpha])=\left[<<a_{11}^{\prime}>,<a_{21}^{\prime}, a_{22}^{\prime}>, \ldots, \neg^{+}<a_{i 1}^{\prime}, \ldots, a_{i i}^{\prime}>, \ldots>\right],
$$

где все конечные последовательности из списка

$$
\begin{gathered}
<a_{11}^{\prime}>,<a_{21}^{\prime}, a_{22}^{\prime}>, \ldots, \\
<a_{(i-1), 1}^{\prime}, \ldots, a_{(i-1),(i-1)}^{\prime}>,<a_{(i+1), 1}^{\prime}, \ldots, a_{(i+1),(i+1)}^{\prime}>, \ldots
\end{gathered}
$$

содержат лишь вхождения $F$, а

$$
\begin{gathered}
W\left(<a_{i 1}^{\prime}, \ldots, a_{i i}^{\prime}>\right)= \\
W\left(<a_{11}>\right)+W\left(<a_{21}, a_{22}>\right)+W\left(<a_{31}, a_{32}, a_{33}>\right)+\ldots= \\
\frac{\eta\left(<a_{i 1}^{\prime}, \ldots, a_{i i}^{\prime}>\right)}{i} .
\end{gathered}
$$

Поэтому:

$$
\begin{gathered}
\varphi\left(\left[<<a_{11}^{\prime}>,<a_{21}^{\prime}, a_{22}^{\prime}>, \ldots, \neg^{+}<a_{i 1}^{\prime}, \ldots, a_{i i}^{\prime}>, \ldots>\right]\right)= \\
\frac{i-\eta\left(<a_{i 1}^{\prime}, \ldots, a_{i i}^{\prime}>\right)}{i}=1-\frac{\eta\left(<a_{i 1}^{\prime}, \ldots, a_{i i}^{\prime}>\right)}{i}= \\
1-W\left(<a_{11}>\right)-W\left(<a_{21}, a_{22}>\right)-W\left(<a_{31}, a_{32}, a_{33}>\right)-\ldots= \\
1-\varphi\left(\left[<<a_{11}>,<a_{21}, a_{22}>,<a_{31}, a_{32}, a_{33}>, \ldots>\right]\right)=\sim \varphi([\alpha]) .
\end{gathered}
$$

Таким образом, первое равенство доказано.

Рассмотрим теперь второе равенство. Выберем для доказательства компоненты $<a_{i 1}^{\prime}, \ldots, a_{i i}^{\prime}>$ и $<b_{i 1}^{\prime}, \ldots, b_{i i}^{\prime}>$ соответствующей последовательности

$$
<<a_{11}^{\prime}>,<a_{21}^{\prime}, a_{22}^{\prime}>, \ldots,<a_{i 1}^{\prime}, \ldots, a_{i i}^{\prime}>\supset^{+}<b_{i 1}^{\prime}, \ldots, b_{i i}^{\prime}>, \ldots>,
$$

удовлетворяющие условиям из определения связки $\rightarrow^{\mathfrak{K}}$ в $\mathfrak{K}$. Такие компоненты существуют в силу определений матриц $\mathfrak{K}, \mathfrak{N}_{i+1}^{\mathrm{E}}$ и того, что два любых рациональных числа могут быть представлены в виде дробей с общим знаменателем.

Возможны два случая. 
1. $W\left(<a_{i 1}^{\prime}, \ldots, a_{i i}^{\prime}>\right) \leq W\left(<b_{i 1}^{\prime}, \ldots, b_{i i}^{\prime}>\right)$. В этом случае очевидно, что правая часть второго равенства есть 1 . Далее:

$$
\begin{gathered}
{[\alpha] \rightarrow^{\mathfrak{K}}[\beta]=} \\
{\left[<<a_{11}^{\prime}>, \ldots,<a_{(i-1), 1}^{\prime}, \ldots, a_{(i-1),(i-1)}^{\prime}>,\right.} \\
<\underbrace{T \ldots T}_{i}>,<a_{(i+1), 1}^{\prime}, \ldots, a_{(i+1),(i+1)}^{\prime}>, \ldots>]= \\
{[<<T>,<F F>,<F F F>, \ldots>] .}
\end{gathered}
$$

Значит, $\varphi\left([\alpha] \rightarrow^{\mathfrak{K}}[\beta]\right)=W(<T>)=1$. Что и дает требующееся доказательство первого случая.

2. $W\left(<a_{i 1}^{\prime}, \ldots, a_{i i}^{\prime}>\right)>W\left(<b_{i 1}^{\prime}, \ldots, b_{i i}^{\prime}>\right)$. Тогда правая часть равенства в силу определения $\varphi$ и $\rightarrow$ равняется $1-W(<$ $\left.a_{i 1}^{\prime}, \ldots, a_{i i}^{\prime}>\right)+W\left(<b_{i 1}^{\prime}, \ldots, b_{i i}^{\prime}>\right)$. Но, согласно определению $\rightarrow^{\mathfrak{K}}$ и $\supset^{+}$, а также поскольку имеет место

$$
R\left(<a_{i 1}^{\prime}, \ldots, a_{i i}^{\prime}>,<b_{i 1}^{\prime}, \ldots, b_{i i}^{\prime}>\right),
$$

то число вхождений $T$ в $<a_{i 1}^{\prime}, \ldots, a_{i i}^{\prime}>\supset^{+}<b_{i 1}^{\prime}, \ldots, b_{i i}^{\prime}>$ есть

$$
\eta\left(<b_{i 1}^{\prime}, \ldots, b_{i i}^{\prime}>\right)+\left(i-\eta\left(<a_{i 1}^{\prime}, \ldots, a_{i i}^{\prime}>\right)\right) .
$$

Значит:

$$
\begin{gathered}
W\left(<a_{i 1}^{\prime}, \ldots, a_{i i}^{\prime}>\supset^{+}<b_{i 1}^{\prime}, \ldots, b_{i i}^{\prime}>\right)= \\
\frac{\eta\left(<b_{i 1}^{\prime}, \ldots, b_{i i}^{\prime}>\right)+\left(i-\eta\left(<a_{i 1}^{\prime}, \ldots, a_{i i}^{\prime}>\right)\right)}{i}= \\
1-\frac{\eta\left(<a_{i 1}^{\prime}, \ldots, a_{i i}^{\prime}>\right)}{i}+\frac{\eta\left(<b_{i 1}^{\prime}, \ldots, b_{i i}^{\prime}>\right)}{i} .
\end{gathered}
$$

То есть $\varphi\left([\alpha] \rightarrow^{\mathfrak{K}}[\beta]\right)$ в этом случае также равняется $1-W\left(<a_{i 1}^{\prime}, \ldots, a_{i i}^{\prime}>\right)+W\left(<b_{i 1}^{\prime}, \ldots, b_{i i}^{\prime}>\right)$. Что дает искомое доказательство второго случая. 
Матрица $\mathfrak{K}$ не является фактор-семантикой для бесконечнозначной логики Лукасевича, однако она имеет локальные черты фактор-семантики, поскольку при определении связки $\rightarrow^{\mathfrak{K}}$ потребовалось использовать отношение $R$ соответствующей конечнозначной фактор-матрицы $\mathfrak{N}_{i+1}^{\mathrm{E}}$. Такая семантика может быть названа локальной фактор-семантикой.

Отметим, что взятие сумм весов конечных $T F$-последовательностей легко распространяется и на соответствующее подмножество элементов матрицы $\bigotimes_{1}^{\infty} \mathfrak{N}_{i+1}^{\mathrm{L}}$. А именно, назовем весом $W^{\prime}$ компонента $\left[\alpha_{j}\right]$ в элементе $<\left[\alpha_{1}\right], \ldots,\left[\alpha_{j}\right], \ldots>$ матрицы $\bigotimes_{1}^{\infty} \mathfrak{N}_{i+1}^{\mathrm{L}}$ значение $W\left(<a_{1}, \ldots, a_{j}>\right)$ при каком-нибудь $<a_{1}, \ldots, a_{j}>$ из $\left[\alpha_{j}\right]$.

Определим теперь множество

$$
B^{\prime} \subset\left\{<\left[\alpha_{1}\right],\left[\alpha_{2}\right], \ldots>:\left[\alpha_{i}\right] \in B^{i} / \cong\right\},
$$

что всякая последовательность $<\left[\alpha_{1}\right],\left[\alpha_{2}\right], \ldots>$ из $\bigotimes_{1}^{\infty} \mathfrak{N}_{i+1}^{\mathrm{E}}$ содержится в $B^{\prime}$, если и только если $\Sigma_{1}^{\infty} W^{\prime}\left(\left[\alpha_{j}\right]\right)$ есть рациональное число из интервала $[0 ; 1]$.

Тогда $<\left[\alpha_{1}\right],\left[\alpha_{2}\right], \ldots>\cong<\left[\beta_{1}\right],\left[\beta_{2}\right], \ldots>$, если и только если:

$$
\Sigma_{1}^{\infty} W^{\prime}\left(\left[\alpha_{i}\right]\right)=\Sigma_{1}^{\infty} W^{\prime}\left(\left[\beta_{j}\right]\right),
$$

где $i$ и $j$ пробегают по всем индексам компонентов $<\left[\alpha_{1}\right],\left[\alpha_{2}\right], \ldots>$ и $<\left[\beta_{1}\right],\left[\beta_{2}\right], \ldots>$, соответственно.

Рассмотрим матрицу

$$
\mathfrak{K}^{\prime}=\left\langle B^{\prime} / \cong, \neg^{\mathfrak{K}^{\prime}}, \rightarrow^{\mathfrak{K}^{\prime}},\{[<[T],[F F],[F F F], \ldots>]\}\right\rangle,
$$

где $B^{\prime} / \cong$ есть результат разбиения множества $B^{\prime}$ по отношению эквивалентности $\cong$, а $[<[T],[F F],[F F F], \ldots>]$ есть класс последовательностей из $B^{\prime}$, эквивалентных последовательности, все компоненты которой, за исключением первого, содержат лишь вхождения $F$, а первый компонент есть $[T]$, то есть класс таких последовательностей $<\left[\alpha_{1}\right],\left[\alpha_{2}\right], \ldots>$, что $\Sigma_{1}^{\infty} W^{\prime}\left(\left[\alpha_{i}\right]\right)=1$.

Связки $\neg^{\mathfrak{K}^{\prime}}$ и $\rightarrow{ }^{\mathfrak{K}^{\prime}}$ определяются следующим образом:

- $\neg^{\mathfrak{K}^{\prime}}\left(\left[<\left[\alpha_{1}\right],\left[\alpha_{2}\right], \ldots>\right]\right)=\left[<\left[\alpha_{1}^{\prime}\right],\left[\alpha_{2}^{\prime}\right], \ldots,\left[\neg^{+} \alpha_{j}^{\prime}\right], \ldots>\right]$, где правая часть равенства означает класс последовательностей, эквивалентных последовательности, в которой все 
компоненты

$$
\left[\alpha_{1}^{\prime}\right],\left[\alpha_{2}^{\prime}\right], \ldots,\left[\alpha_{j-1}^{\prime}\right],\left[\alpha_{j+1}^{\prime}\right], \ldots
$$

содержат лишь вхождения $F$, а $\left[\alpha_{j}^{\prime}\right]$ таково, что $W^{\prime}\left(\left[\alpha_{j}^{\prime}\right]\right)=$ $\Sigma_{1}^{\infty} W^{\prime}\left(\left[\alpha_{i}\right]\right)$, где $i$ пробегает по всем индексам компонентов последовательности $<\left[\alpha_{1}\right],\left[\alpha_{2}\right], \ldots>$.

$$
\begin{gathered}
{\left[<\left[\alpha_{1}\right],\left[\alpha_{2}\right], \ldots>\right] \rightarrow \rightarrow^{\mathfrak{K}^{\prime}}\left[<\left[\beta_{1}\right],\left[\beta_{2}\right], \ldots>\right]=} \\
{\left[<\left[\alpha_{1}^{*}\right],\left[\alpha_{2}^{*}\right], \ldots,\left[\alpha_{j}^{*} \supset^{+} \beta_{j}^{*}\right], \ldots>\right],}
\end{gathered}
$$

где правая часть равенства означает класс последовательностей, эквивалентных последовательности, в которой все компоненты

$$
\left[\alpha_{1}^{*}\right],\left[\alpha_{2}^{*}\right], \ldots,\left[\alpha_{j-1}^{*}\right],\left[\alpha_{j+1}^{*}\right], \ldots
$$

содержат только вхождения $F$, а $\left[\alpha_{j}^{*}\right]$ и $\left[\beta_{j}^{*}\right]$ таковы, что $W^{\prime}\left(\left[\alpha_{j}^{*}\right]\right)=\Sigma_{1}^{\infty} W^{\prime}\left(\left[\alpha_{i}\right]\right), W^{\prime}\left(\left[\beta_{j}^{*}\right]\right)=\Sigma_{1}^{\infty} W^{\prime}\left(\left[\beta_{i}\right]\right)$, где $i$ пробегает по всем индексам компонентов последовательностей $<\left[\alpha_{1}\right],\left[\alpha_{2}\right], \ldots>$ и $<\left[\beta_{1}\right],\left[\beta_{2}\right], \ldots>$, соответственно; а также в фактор-матрице $\mathfrak{N}_{j+1}^{\mathrm{E}}$ соответствующей конечнозначной логики Лукасевича выполняется $R\left(\alpha_{j}^{*}, \beta_{j}^{*}\right)$.

Матрица $\mathfrak{K}^{\prime}$ также изоморфна матрице $\mathfrak{M}_{\infty}^{\mathrm{L}}$ с рациональнозначным множеством-носителем. Докажем соответствующую теорему.

TEOPEмA 2. Матрица $\mathfrak{K}^{\prime}$ изоморфна матрице $\mathfrak{M}_{\infty}^{E}=\langle[0 ; 1], \sim, \rightarrow,\{1\}\rangle$, где $[0 ; 1] \in \mathcal{P}(\mathbb{Q})$.

ДокАзАтельство. Определим отображение $\varphi: B^{\prime} / \cong \mapsto[0 ; 1]$, что для всякого $\left[<\left[\alpha_{1}\right],\left[\alpha_{2}\right]>, \ldots\right]$ в $B^{\prime} / \cong$ выполняется:

$$
\varphi\left(\left[<\left[\alpha_{1}\right],\left[\alpha_{2}\right], \ldots>\right]\right)=\Sigma_{1}^{\infty} W^{\prime}\left(\left[\alpha_{i}\right]\right),
$$

где $i$ пробегает по всем индексам соответствующих «внутренних» классов эквивалентности из $\left[\alpha_{1}\right],\left[\alpha_{2}\right], \ldots$

Данное отображение в силу определения множества $B^{\prime} / \cong$ является взаимнооднозначным. Покажем теперь, что изоморфизм имеет место, то есть выполняются два равенства: 
- $\varphi\left(\neg^{\mathfrak{K}^{\prime}}\left(\left[<\left[\alpha_{1}\right],\left[\alpha_{2}\right], \ldots>\right]\right)\right)=\sim \varphi\left(\left[<\left[\alpha_{1}\right],\left[\alpha_{2}\right], \ldots>\right]\right)$

- $\varphi\left(\left[<\left[\alpha_{1}\right],\left[\alpha_{2}\right], \ldots>\right] \rightarrow^{\mathfrak{K}^{\prime}}\left[<\left[\beta_{1}\right],\left[\beta_{2}\right], \ldots>\right]\right)=$

$$
\varphi\left(\left[<\left[\alpha_{1}\right],\left[\alpha_{2}\right], \ldots>\right]\right) \rightarrow \varphi\left(\left[<\left[\beta_{1}\right],\left[\beta_{2}\right], \ldots>\right]\right) .
$$

Рассмотрим первое из этих двух равенств. Верно следующее:

$$
\neg^{\mathfrak{K}^{\prime}}\left(\left[<\left[\alpha_{1}\right],\left[\alpha_{2}\right], \ldots>\right]\right)=\left[<\left[\alpha_{1}^{\prime}\right],\left[\alpha_{2}^{\prime}\right], \ldots,\left[\neg^{+} \alpha_{j}^{\prime}\right], \ldots>\right],
$$

где стоящее справа выражение удовлетворяет условиям определения $\neg^{\mathfrak{K}^{\prime}}$.

Тогда:

$$
\begin{gathered}
\varphi\left(\left[<\left[\alpha_{1}^{\prime}\right],\left[\alpha_{2}^{\prime}\right], \ldots,\left[\neg^{+} \alpha_{j}^{\prime}\right], \ldots>\right]\right)= \\
W^{\prime}\left(\left[\neg^{+} \alpha_{j}^{\prime}\right]\right)=1-W^{\prime}\left(\left[\alpha_{j}^{\prime}\right]\right)=1-\Sigma_{1}^{\infty} W^{\prime}\left(\left[\alpha_{i}\right]\right)= \\
1-\varphi\left(\left[<\left[\alpha_{1}\right],\left[\alpha_{2}\right], \ldots>\right]\right)=\sim \varphi\left(\left[<\left[\alpha_{1}\right],\left[\alpha_{2}\right], \ldots>\right]\right) .
\end{gathered}
$$

Что и дает искомое доказательство первого равенства.

Рассмотрим второе равенство. Выберем для доказательства классы эквивалентности $\left[\alpha_{j}^{*}\right]$ и $\left[\beta_{j}^{*}\right]$, удовлетворяющие условиям из определения связки $\rightarrow \rightarrow^{\mathfrak{K}^{\prime}}$ в $\mathfrak{K}^{\prime}$. Такие классы существуют в силу определений матриц $\mathfrak{K}^{\prime}, \mathfrak{N}_{j+1}^{\mathrm{L}}$ и того, что два любых рациональных числа могут быть представлены в виде дробей с общим знаменателем.

Возможны два случая.

1. $W\left(\left[\alpha_{j}^{*}\right]\right) \leq W\left(\left[\beta_{j}^{*}\right]\right)$. В этом случае очевидно, что правая часть второго равенства есть 1. Далее:

$$
\begin{gathered}
\varphi\left(\left[<\left[\alpha_{1}\right],\left[\alpha_{2}\right], \ldots>\right] \rightarrow^{\mathfrak{K}^{\prime}}\left[<\left[\beta_{1}\right],\left[\beta_{2}\right], \ldots>\right]\right)= \\
W\left(\left[\alpha_{j}^{*} \supset^{+} \beta_{j}^{*}\right]\right)=1,
\end{gathered}
$$

что дает искомое доказательство данного случая. 
2. $W\left(\left[\alpha_{j}^{*}\right]\right)>W\left(\left[\beta_{j}^{*}\right]\right)$. Тогда правая часть второго равенства в силу определения $\varphi$ и $\rightarrow$ есть $1-W\left(\left[\alpha_{j}^{*}\right]\right)+W\left(\left[\beta_{j}^{*}\right]\right)$. Левая же часть второго равенства есть в этом случае:

$$
\frac{j-\eta\left(\alpha_{j}^{*}\right)+\eta\left(\beta_{j}^{*}\right)}{j}=1-W\left(\left[\alpha_{j}^{*}\right]\right)+W\left(\left[\beta_{j}^{*}\right]\right),
$$

что завершает доказательство теоремы.

Полученная матрица $\mathfrak{K}^{\prime}$ также имеет локальные черты фактор-семантики и может рассматриваться в качестве локальной фактор-семантики для $\mathrm{E}_{\infty}$. Более того, процедуру построения матрицы $\mathfrak{K}^{\prime}$ можно рассматривать в качестве особой операции над логическими матрицами, которая может быть применена и к более широкому классу логик.

Отношение изоморфизма логических матриц обладает следующим свойством:

$$
\mathfrak{M}_{1} \equiv \mathfrak{N} \& \mathfrak{M}_{2} \equiv \mathfrak{N} \Rightarrow \mathfrak{M}_{1} \equiv \mathfrak{M}_{2}
$$

Поэтому теоремы 1 и 2 позволяют вывести следствие.

СлЕДСтвиЕ 1. Матрица $\mathfrak{K}$ изоморфна матрице $\mathfrak{K}^{\prime}$.

ДокАЗАТЕЛьСтво. Доказательство непосредственно следует из теорем 1 и 2 и вышеприведенного свойства отношения изоморфизма логических матриц.

Возвращаясь к поставленным в начале статьи вопросам, можно заключить, что бесконечнозначные логики, совпадающие по классу тавтологий с пересечением классов тавтологий некоторой бесконечной последовательности конечнозначных логик, каждая из которых имеет фактор-семантику, могут иметь «локальные фактор-семантики», построенные с использованием процедур, аналогичных рассмотренным в теоремах 1 и 2 . Причем, результирующие матрицы будут изоморфны исходным бесконечнозначным логическим матрицам. 


\section{Литература}

[1] Карпенко А.C. Развитие многозначной логики. М.: Издательство ЛКИ, 2010.

[2] Карпенко А.С. Логики Лукасевича и простые числа. М.: Наука, 2000.

[3] Karpenko A.S. Factor-Semantics for n-valued Logics // Studia Logica. 42(2/3). 1983. P. 179-185.

[4] Rescher N. Many-Valued Logic. New York: McGraw Hill, 1969.

\section{References (transliteration)}

[1] Karpenko A.S. Razvitie mnogoznachnoj logiki. M.: Izdatel'stvo LKI, 2010.

[2] Karpenko A.S. Logiki Lukasevicha i prostye chisla. M.: Nauka, 2000.

[3] Karpenko A.S. Factor-Semantics for n-valued Logics // Studia Logica. 42(2/3). 1983. P. 179-185.

[4] Rescher N. Many-Valued Logic. New York: McGraw Hill, 1969. 